\title{
ORIGINALS
}

\section{Demonstration of Anti-“a-component” Antibody - A Possible Means to Differentiate Patients with Auto-Antibodies to Endogenous Insulin from Insulin-Treated Patients}

\author{
S. Kawazu, Y. Kanazawa, H. Kajinuma, E. Miki, T. Kuzuya* and K. Kosaka \\ The Third Dept. of Internal Medicine, Faculty of Medicine, University of Tokyo, Hongo, Tokyo and \\ *Dept. of Medicine, Jichi Medical School, Minami-kawachimachi, Tochigi-ken, Japan \\ Received: August 16, 1974, and in revised form: December 4, 1974
}

\begin{abstract}
Summary. The presence of anti-“a-component" antibody was examined in sera of 4 groups of patients with or without anti-insulin antibody, using 125I-a-component and the polyethylene glycol precipitation method. ${ }^{125} \mathrm{I}$-a-component crossreacted with insulin antibody. This cross-reactivity was abolished after preincubation of these sera with monocomponent insulin. The specific anti-"a-component" antibody could be estimated in this procedure. After preincubation with monocomponent insulin, significant binding of $125 \mathrm{I}$-a-component was demonstrated in sera of most patients treated with ordinary commercial insulin, but not in sera of 2 hypoglycemic patients suspected of an insulin autoimmune syndrome. Some
\end{abstract}

cases treated with commercial insulin for less than one year and all cases treated with monocomponent insulin for $7-10$ months did not have significant anti-"a-component" antibody. The test for the presence of anti-"a-component" antibody is not definitive but if positive it differentiates "auto-antibodies" from the antibodies produced by injections of commercial insulin.

Key words: Anti-a-component antibody, anti-insulin antibody, insulin autoimmune syndrome, hypoglycemia, monocomponent insulin.
Since the first report by Hirata et al. [1] in 1970, case reports of patients having insulin antibodies, with no previous history of insulin injections, have been accumulated [2-8]. However, it is impossible to prove that a patient has never received insulin and it is therefore desirable to find some objective method to differentiate autoantibodies from antibodies produced by repeated injections of exogenous insulin $[3-6]$.

Most insulin preparations commercially available are known to contain some impurities with higher molecular weight. They were designated as a-, b-, and c-components by the order of appearance when fractionated by gel filtration [9]. They are more immunogenic by themselves than pure insulin, which is the main constituent of c-component, and proved to be responsible for the induction of anti-insulin antibody after long-term insulin treatment [9-11].

We contrived a method to estimate specific anti"a-component" antibody using ${ }^{125} \mathrm{I}$-a-component and a polyethylene glycol precipitation method. We estimated anti-a-component antibody in 4 diabetic groups with or without anti-insulin antibody and tried to differentiate the hypoglycemic patients suspected of insulin autoimmune syndrome from the patients treated with "impure" commercial insulin.

\section{Materials and Methods}

Sera were collected from: (a) 38 diabetic patients receiving injections of conventional commercial insulins of intermediate-type for different periods in our clinic and bearing anti-insulin antibody in various amounts, (b) 4 diabetics started on treatment with monocomponent-insulin (MC-insulin, Novo) 7 to 10 months before collection of the sera, (c) 10 diabetic patients treated by diet or oral drugs, not by insulin injections, all of whom had no anti-insulin antibody, and (d) 2 hypoglycemic patients suspected of having "autoimmune" anti-insulin antibody, whose case records are summarized as follows.

\section{Case 1}

K.M., a 59 year old Japanese male, was admitted because of a hypoglycemic attack. He had a gastrectomy for duodenal ulcer at the age of 56. The next year, he started to have repeated episodes of cold sweating, palpitation and collapse, which were improved by food intake. Such episodes occurred mainly in the evening before supper, associated with numbness and involuntary movement of head and facial muscles. He had no family history of diabetes mellitus and no past history of insulin injections. On 
admission, physical examinations of chest and the abdomen were within normal limits. Neurological examination revealed the disturbances mentioned above even when normoglycemic, but these findings gradually disappeared thereafter.

Urinalysis, blood counts and cerebrospinal fluid analysis were all normal. Function tests of the liver, the kidney and the exocrine pancreas were within normal limits. Results of endocrinological tests of the hypophysis, the thyroid, the parathyroid and the adrenals were also in the normal range. Blood glucose values during $100 \mathrm{~g}$ oral GTT were $68,280,239,137$, 97 and $30 \mathrm{mg} / 100 \mathrm{ml}$ at $0,30,60,90,120$ and 180 min after glucose load. His consciousness became disturbed at $180 \mathrm{~min}$. Immunoreactive insulin levels in untreated serum were determined, by a double antibody method [12], to be $773 \mu \mathrm{U} / \mathrm{ml}$ in the fasting state.

Tolbutamide test, L-leucine loading, injection of glucagon and long-term fasting test (3 days) were carried out, but the hypoglycemic attack was not induced. Angiographic and scintigraphic examinations of the pancreas and X-ray examinations of the pancreatic ducts revealed no abnormal findings. Finally, insulin binding proteins were proved to be present in his serum by incubating the serum with ${ }^{125} \mathrm{I}$-insulin and subsequent precipitation by polyethylene glycol (M.W. 6,000, final concentration 12.5\%) [13, 14]. Furthermore, this binding protein was demonstrated to belong to IgG class immunoglobulin with light chain of $\mathrm{K}$-type only, by the double-antibody method and by immunoelectrophoresis using specific antisera directed to each class of human immunoglobulins and light chains. ${ }^{125} \mathrm{I}$-insulin bound to serum protein was dissociated by Sephadex G-75 gel filtration at pH 2.8.

\section{Case 2}

C.I., a 77 year old Japanese male was admitted because of repeated attacks of disturbance of consciousness, which were relieved by meals. During the past two years, he occasionally felt dizzy before supper. On April 30, 1972, he was found in a delirious state, moving upper and lower extremities, at midnight. He recovered spontaneously with profuse sweating and did not remember what happened. Such attacks occurred more frequently after May, 1972 and were relieved by meals or glucose injections. He had suffered from pulmonary tuberculosis ten years and from stomach ulcer 3 years previously, but had no history of surgical operations or insulin injections. Family history for diabetes mellitus was negative. On physical examination, there were essentially no abnormalities except slight anemia. He was not obese. Neurological examinations were normal.
Blood glucose levels after $50 \mathrm{~g}$ oral glucose load were $64,166,209,208,128$ and $37 \mathrm{mg} / 100 \mathrm{ml}$ at 0 , $30,60,90,120$ and $180 \mathrm{~min}$, respectively. Radioimmunoassay of insulin by a double antibody method using the untreated sera gave extremely high values of $2500-7000 \mu \mathrm{U} / \mathrm{ml}$. Laboratory examination revealed no other endocrinological or metabolic abnormalities. Subsequent study of sera by the ethanol precipitation method and Sephadex G-50 gel filtration revealed that insulin-binding protein was present in his serum; it migrated at the $\gamma$-globulin region on agar plate electrophoresis. ${ }^{125}$ I-insulin bound to this fraction was dissociated at $\mathrm{pH} 3.0$.

Methods for the estimation of antibodies:

${ }^{125} \mathrm{I}$-insulin and ${ }^{125} \mathrm{I}$-a-component were prepared by the method of Hunter and Greenwood employing crystalline pork insulin or beef a-component, which was kindly supplied from Novo Research Institute (Copenhagen). Labelled a-component was purified using a Sephadex G-75 column $(1 \times 20 \mathrm{~cm})$. The first radioactive peak was pooled and kept frozen until use. For the detection and estimation of antibodies, the polyethylene glycol (PEG, M.W. 6,000) method was employed $[12,13]$.

The mixture of $50 \mu \mathrm{l}$ sample serum, $300 \mu \mathrm{l} 0.2 \mathrm{M}$ borate buffer ( $\mathrm{pH} 8.4$ ) and $50 \mu \mathrm{l}$ 125I-insulin $(1 \mathrm{ng} / \mathrm{ml})$ or ${ }^{125} \mathrm{I}$-a-component $(1 \mathrm{ng} / \mathrm{ml})$ was incubated for 48 hrs at $4{ }^{\circ} \mathrm{C}$. To separate bound and free, $400 \mu \mathrm{l} 25 \%$ PEG was added and the mixture was centrifuged at $3,000 \mathrm{rpm}$ for $45 \mathrm{~min}$. Supernatant was discarded and the precipitate counted in a well-type scintillation spectrometer. Preliminary experiments revealed that the final concentration of $12.5 \%$ PEG was suitable to separate bound and free for both insulin and a-component.

\section{Results}

Antibodies produced by repeated injections of acomponent are known to cross-react with insulin [10, 11]. In our hands, all sera containing insulin antibody bound ${ }^{125} \mathrm{I}$-a-component significantly. To eliminate this cross-reaction, unlabelled MC-insulin (Novo) was added to sera prior to the addition of ${ }^{225} \mathrm{I}$-a-component. Various amounts of $\mathrm{MC}$-insulin (beef: pork = $1: 1$, dissolved in $100 \mu \mathrm{l}$, was added to this mixture of $50 \mu \mathrm{l}$ serum and $200 \mu \mathrm{l}$ borate buffer, and preincubated for $48 \mathrm{hrs}$ at $4^{\circ} \mathrm{C}$. Thereafter, $50 \mu 1{ }^{125}$ I-insulin $(1 \mathrm{ng} / \mathrm{ml})$ or ${ }^{125} \mathrm{I}$-a-component $(1 \mathrm{ng} / \mathrm{ml})$ was added and incubated for a further $48 \mathrm{hrs}$ at $4^{\circ} \mathrm{C}$ and then bound and free were separated by the PEG method. Fig. 1 shows the data of ${ }^{125} \mathrm{I}$-a-component binding of sera following preincubation with $\mathrm{MC}$-insulin. After preincubation with $\mathrm{MC}$-insulin, binding 


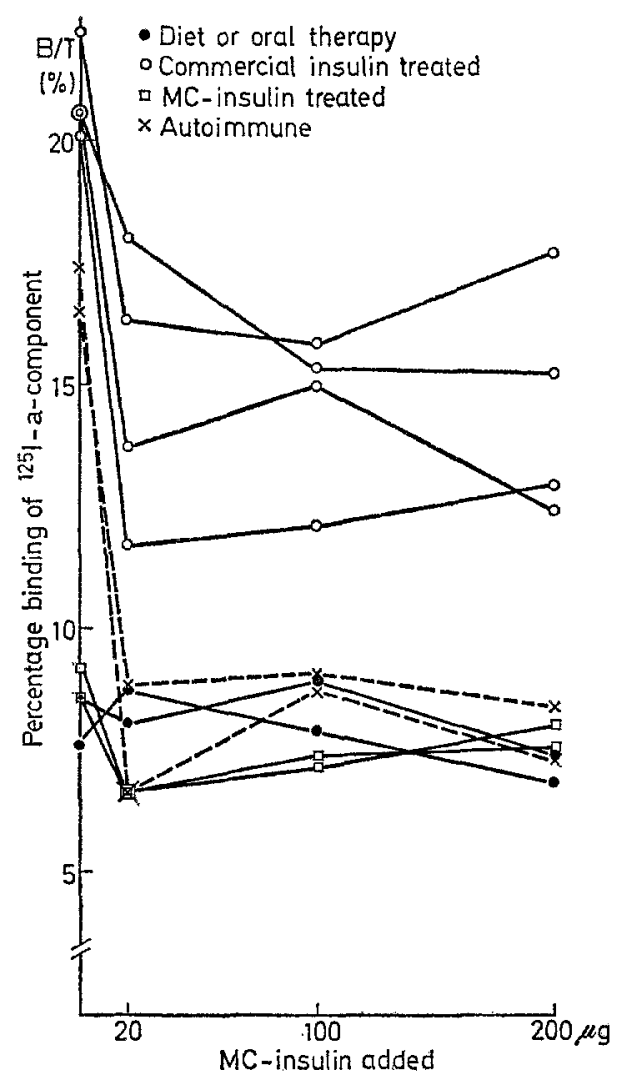

Fig. 1. Effect of unlabelled MC-insulin (beef : pork $=1: 1$ ) on the binding of ${ }^{125} \mathrm{I}$-a-component

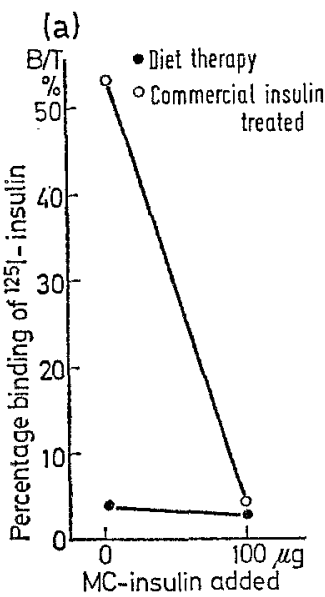

(b)

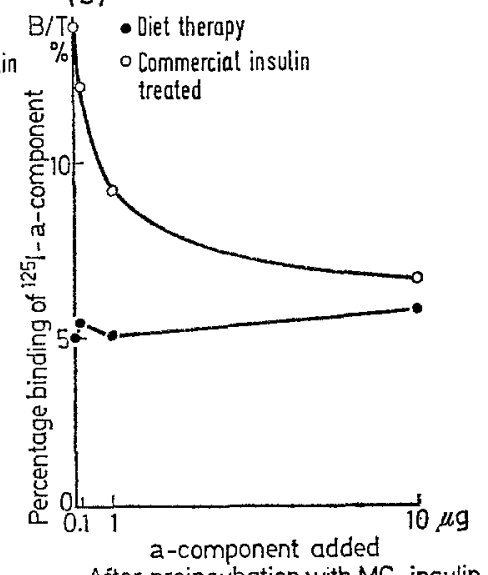

Effect of preincubation

After preincubation with $M C$-insulin

Fig. 2a. Effect of preincubation with MC-insulin $(100 \mu \mathrm{g})$ on the binding of 125I-insulin of a commercial insulin (not highly purified) treated patient and a control patient

Fig. 2b. After preincubation with MC-insulin (100 $\mu \mathrm{g})$, though there exists no significant binding of ${ }^{125}$ I-insulin (Fig. 2-a), specific binding of ${ }^{125} \mathrm{I}$-a-component persist and is displaced by unlabelled a-component

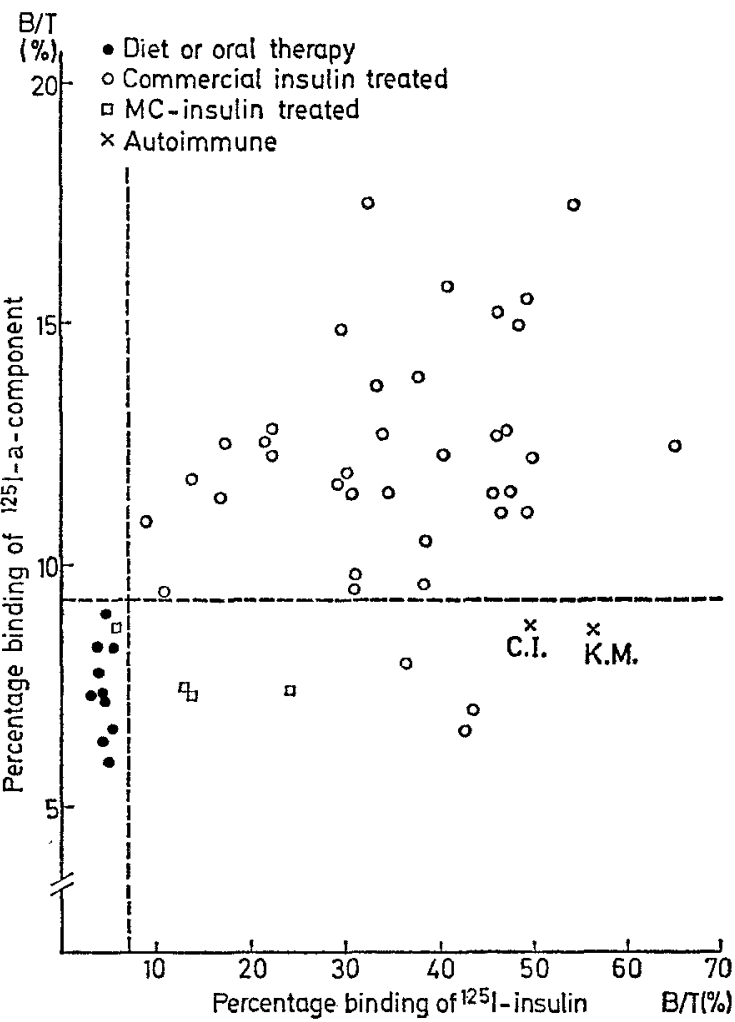

Fig. 3. Binding of ${ }^{125} \mathrm{I}$-insulin versus specific binding of ${ }^{125} \mathrm{I}-\mathrm{a}-$ component in all patients tested

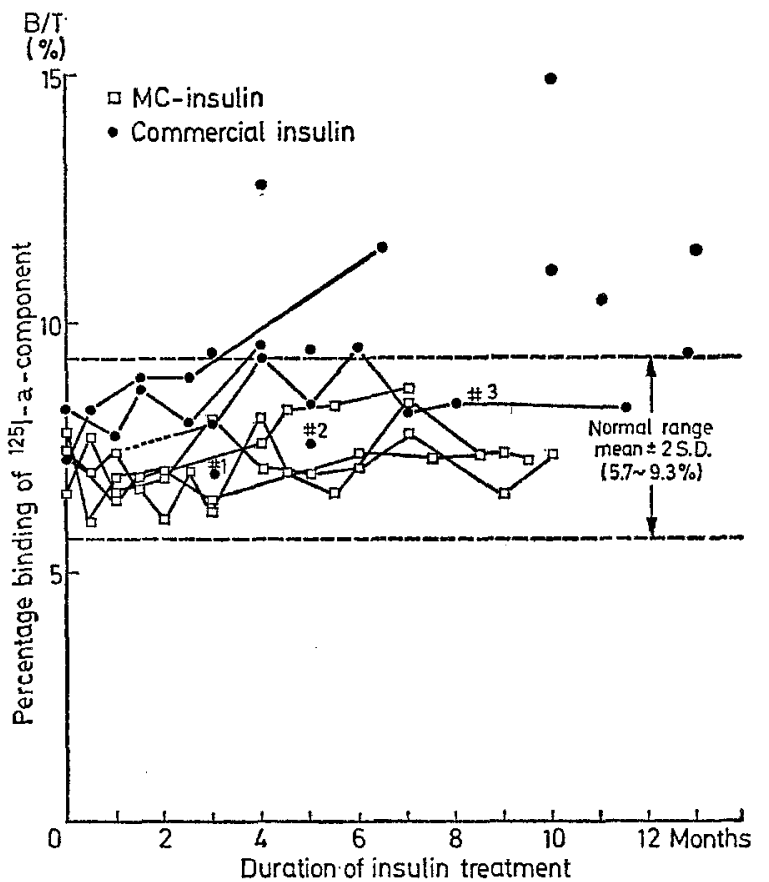

Fig. 4. Appearance of specific binding of ${ }^{125} \mathrm{I}-\mathrm{a}$-component within one year. 3 of the commercial insulin (not highly purified) treated patients have no significant binding of ${ }^{25} \mathrm{I}$-acomponent. $(\# 1,2,3)$ 
of ${ }^{125} \mathrm{I}$-a-component was still present in all sera of insulin-treated patients. In contrast, 2 sera of "autoimmune" patients decreased their binding of ${ }^{125} \mathrm{I}-\mathrm{a}$ component to the control level after preincubation with $20 \mu \mathrm{g}$ or more MC-insulin. As shown in Fig. 2a, the binding of ${ }^{125} \mathrm{I}$-insulin of a serum of an insulintreated patient was completely abolished by this preincubation, but a significant binding of ${ }^{125} \mathrm{I}-\mathrm{a}-\mathrm{com}-$ ponent still persisted. This remaining "specific" binding of ${ }^{125} \mathrm{I}$-a-component was displaced by the addition of unlabelled a-component (Fig. 2b).

The data concerning binding of ${ }^{125}$ I-insulin and ${ }^{125}$ Ia-component of all the cases are presented in Fig. 3 . Sera of most patients treated with commercial insulin bound both ${ }^{225} \mathrm{I}$-insulin and ${ }^{125} \mathrm{I}$-a-component to a greater extent than did sera of control or MC-insulintreated patients. Sera of 3 patients who were treated with commercial insulin for less than one year, however, as well as the sera of 2 "autoimmune" patients, did not bind ${ }^{125} \mathrm{I}$-a-component in spite of a fairly high capacity to bind ${ }^{125}$ I-insulin.

The binding of ${ }^{125} \mathrm{I}$-a-component was related to the duration of insulin treatment (Fig. 4). MC-insulin treatment of 7-10 months did not induce any significant a-component antibody. More than half of the patients treated with commercial insulin showed antia-component antibody within one year, sometimes within 3 months. All cases treated for more than one year had anti-a-component antibody.

\section{Discussion}

It is known that a-component and other impurities with high molecular weight of commercial insulin, induce formation of antibody not only to insulin but also to high molecular weight protein itself $[9,11]$. Schlichtkrull et al. demonstrated anti-a-component antibody in rabbits immunized with a solution of acomponent and in patients treated with commercial insulin [10]. We could also demonstrate "specific" anti-a-component antibody after abolition of insulin binding by preincubation with pure insulin (MC-insulin, Novo).

All the commercial insulins of intermediate or long-acting type available in Japan contain beef insulin. Consequently, they probably contain beef acomponent as impurities. In view of the report that acomponent is more immunogenic than insulin itself, it is likely that Japanese diabetic patients treated with commercial insulin possess anti-a-component antibody in addition to anti-insulin antibody. Indeed, we were able to demonstrate significant ${ }^{125}$ I-a-component binding in their sera after preincubation with $\mathrm{MC}$-in- sulin, except for 3 patients who had been treated for less than one year. On the other hand, none of 4 patients treated with MC-insulin had anti-a-component antibody.

The "insulin autoimmune syndrome" has not been firmly established as a clinical entity despite more than 10 case reports $[1-8]$ of patients with anti-insulin antibody in the absence of previous history of insulin injections, mainly due to the difficulty of proving that the patients had never received insulin. A few suggestive data have been obtained to differentiate so-called autoantibody from antibody produced by exogenous insulin injections $[3,4,5]$. "Autoantibody" to insulin was shown to belong almost exclusively to $\mathrm{IgG}$, and that their light chains are of $\mathrm{K}$-type. In contrast, the sera of insulin-treated patients contain insulin antibodies of IgG, IgM and IgA classes, and their light chains consist of both $\mathrm{K}$ - and L-types [4, 15]. In this respect, our first case suspected of insulin autoimmunity conforms with previous reports.

Furthermore, the sera of 2 patients suspected of the insulin autoimmune syndrome showed significant binding of ${ }^{125} \mathrm{I}$-insulin, but not of ${ }^{125} \mathrm{I}$-a-component after preincubation with MC-insulin. This showed the distinctive contrast between autoantibody and antibody induced by injections of commercial insulin. However, the lack of a-component binding does not completely rule out the possibility of previous treatment with commercial insulin. The patients treated with commercial insulin for less than one year often did not develop significant a-component antibody. It is also possible that anti-a-component antibody may disappear more rapidly than anti-insulin antibody after the cessation of insulin treatment. The pure insulin preparation ( $\mathrm{MC}$-insulin) did not induce a significant amount of anti-a-component antibody. Thus, it is still difficult to evaluate the significance of the absence of anti-a-component antibody.

Despite these limitations, the examination of $125 \mathrm{I}-$ a-component after preincubation with MC-insulin seems to provide an additional means of differentiating "autoimmune antibody" from antibody produced by exogenous injections of commercial insulin. At least, if significant a-component binding is detected, it rules out insulin auto-immunity and suggests a history of insulin injections.

Acknowledgements. We thank Dr. Y. Hirata for sending us beef a-component (Novo). Monocomponent insulin was kindly donated from Novo Research Institute through Kodama Co. Ltd. We are grateful to Drs G. Inoue, T. Sawada and T. Serizawa (Faculty of Geriatrics, University of Tokyo) and Drs A. Matsuda and T. Sakata (Kantochuo-hospital, Tokyo) for permitting us to study the sera of patients of "insulin autoimmune syndrome" and allowing us to publish their case history. 


\section{References}

1. Hirata, Y., Ishizu, H., Ouchi, N., Motomura, S., Abe, M., Hara, Y., Wakasugi, H., Takahashi, I., Takano, H., Tana. ka, M., Kawano, H., Kanesaki, T.: Insulin autoimmunity in a case with spontaneous hypoglycemia. J. Japan Diab. Soc. 13, 312-319 (1970)

2. Hirata, Y., Arimachi, M.: Insulin autoimmune syndromethe second case. J. Japan Diab. Soc. 15, 187-192 (1972)

3. Følling, I., Norman, N.: Hyperglycemia, hypoglycemic attacks, and production of anti-insulin antibodies without previous known immunization. Immunological and functional studies in a patient. Diabetes 21, 814-826 (1972)

4. Nakagawa, S., Suda, S., Kudo, M., Kawasaki, M.: A new type of hypoglycemia in a new born infant. Diabetologia 9, 367-375 (1973)

5. Ohneda, A., Matsuda, K., Sato, M., Yamagata, S., Sato, T.: Hypoglycemia due to apparent antibodies to insulin. Characterization of insulin-binding protein. Diabetes 23, $41-50$ (1974)

6. Hirata, Y., Nishimura, H., Tominaga, M., Oguchi, T., Nakamura, Y.: Insulin autoimmunity in a case with mild diabetes. The third case of insulin-autoimmune syndrome. J. Japan Diab. Soc. 15, 336 - 342 (1972)

7. Sato, T., Saito, T., Honma, K., Ono, H., Yasuda, K. Torikai, T.: A case with considerable hyperinsulinemia and decreased glucose tolerance. J. Japan Diab. Soc. 15 (Suppl.), 176 (1972)

8. Nakano, M., Ito, M., Yamamoto, T., Hamazaki, T.: A case of insulin autoimmune syndrome followed-up with steroid therapy. J. Japan Diab. Soc. 17 (Suppl., 143 (1974)
9. Schlichtkrull, J., Brange, J., Christiansen, Aa. H., Hailund, O., Heding, L. G., Jörgensen, K. H.: Clinical aspects of insulin-antigenicity. Diabetes 21 (Suppl. 2), 649-656 (1972)

10. Schlichtkrull, J.: Monocomponent insulin and its clinical implications. Manuscript of paper to be read to the insulin study circle, Tokyo Oct., 1973

11. Schlichtkrull, J.: Antibodies to components of insulin. Lecture held at the Royal Society of Medicine's Meeting, London Feb., 1974

12. Morgan, C. R., Lazarow, A.: Immunoassay of insulin: Two antibody system. Plasma insulin levels of normal, subdiabetic and diabetic rats. Diabetes 12, 115-126 (1963)

13. Desbuquois, B., Aurbach, G. D.: Use of polyethylene glycol to separate free and antibody-bound peptide hormones in radioimmunoassays. J. clin. Endocr. 33, 732-738 (1971)

14. Nakagawa, S., Nakayama, H., Sasaki, T., Yoshino, K., Yu, Y. Y., Shinozaki, K., Aoki, S., Mashimo, K.: The determination of serum free insulin levels in the insulin-treated patients. J. Japan Diab. Soc. 15, 403-408 (1972)

15. Kenbo, T., Hirata, Y.: Immunological study of insulin antibodies in the sera of 14 cases of insulin autoimmune syndrome. J. Japan Diab. Soc. 17 (Supp1.), 145 (1974)

Dr. S. Kawazu

The Third Dept. of Internal Medicine

Faculty of Medicine

University of Tokyo

Hongo, Tokyo

Japan 